\title{
In-situ TEM Study of Oxygen Surface Exchange on Ceria, Gd-doped Ceria and Pr- doped Ceria
}

Mai $\operatorname{Tan}^{1}$, Peter Crozier ${ }^{2}$ and Joshua Vincent ${ }^{3}$

${ }^{1}$ Arizona State University, CHANDLER, Arizona, United States, ${ }^{2}$ Arizona State University, Tempe, Arizona, United States, ${ }^{3}$ Arizona State University, United States

Oxygen exchange reactions play an important role in many energy conversion applications. For example, in solid oxide fuel cells (SOFC), at the cathode interface, molecular oxygen is first reduced to oxygen ions and then diffuses through a solid electrolyte to interact on the anode side for fuel oxidation [1]. Oxygen exchange reactions are complex and consist of multiple steps including $\mathrm{O} 2$ absorption/desorption, molecular dissociation/association, electron transfer, and incorporation/removal oxygen into or from surface oxygen vacancies. Nonstoichiometric oxides, such as $\mathrm{CeO} 2$ and ceria-based doped materials, are ideal candidates for oxygen exchange applications, because of the ability of reversibly exchange lattice oxygen with the ambient environment while maintaining a stable structure [2]. Doping ceria with aliovalent cations, such as Gd and Pr, can significantly increase the oxygen vacancy concentration resulting in higher ionic conductivity [3]. Due to the complexity of the multi-step oxygen exchange reaction, the surface exchange properties and local electronic structure with different dopants are not well understood. To develop a deeper understanding of the materials, it is desirable to determine the oxygen surface exchange mechanisms that limit the exchange rate.

Cube shaped 15\% Gd-doped ceria (GDC), 15\% Pr-doped ceria (PDC) and pure ceria (CeO2) were synthesized with a hydrothermal method [3]. Time resolved in-situ aberration-corrected TEM was used to observe atomic level variations in the oxygen vacancy creation/annihilation activities on the material surfaces. The local oxygen vacancy activity can be associated with fluxional behavior of the adjacent locations [4]. Images were acquired on JEOL 2010F and FEI Titan ETEM 80-300 microscope using Gatan $\mathrm{K} 3$ direct electron detector.

Figure 1 shows TEM images of $15 \%$ Gd-doped ceria, 15\% Pr-doped ceria and ceria surfaces in the [110] projection with a $1 \mathrm{~s}$ exposure time at room temperature. All three materials showed a sawtooth-like (111) nano-facet on the (110) surfaces. (111) nano-facet formation was associated with surface reconstruction to lower surface energy giving enhancing stability. Figure 2 shows an integrated 1s AC-TEM image and time sequence images of the step edge of $\mathrm{CeO} 2$ (110) surface in [110] projection at room temperature. One important observation is that the top-surface Ce column is more diffused than sub-surface Ce column, especially at the step edge site. As the dynamic motion of the step edge cation is highlighted in red arrows, the Ce column has shifted $60 \mathrm{pm}$ from time $0 \mathrm{~ms}$ to $130 \mathrm{~ms}$ and shifted back $70 \mathrm{pm}$ at $260 \mathrm{~ms}$. This large cation column relaxation is the result of oxygen exchange between lattice and ambient environment. Further in-situ TEM study will continue on GDC and PDC to exam the dopant effect on surface oxygen exchange mechanisms. 

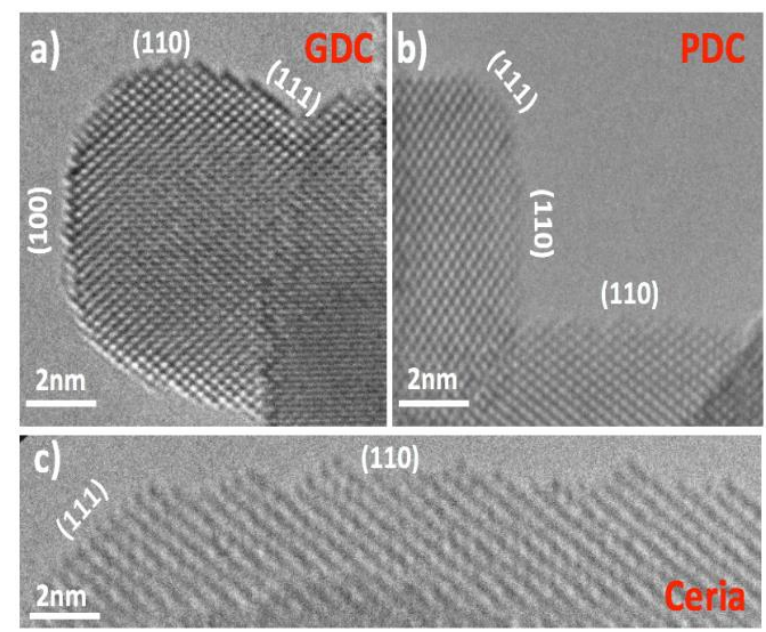

Figure 1. Figure 1. TEM image of a) $15 \%$ Gd-doped ceria b) $15 \%$ Pr-doped ceria and 3) pure ceria surfaces in [110] projection with 1s exposure time at room temperature.

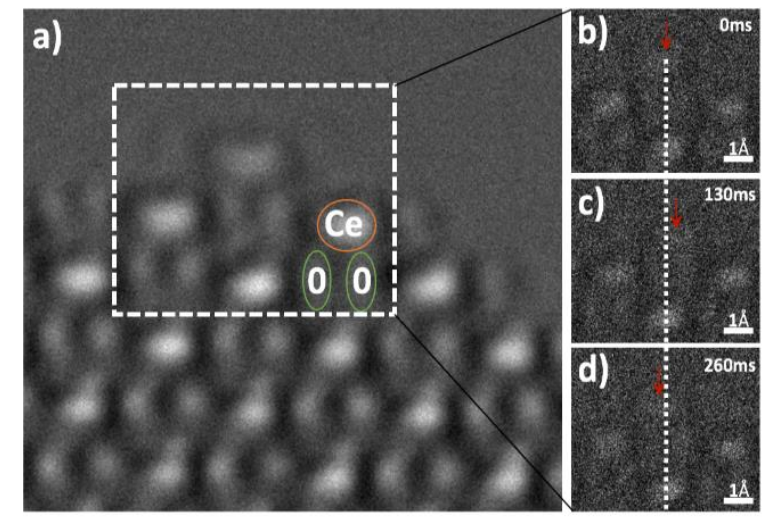

Figure 2. Figure 2. a) integrated 1s AC-TEM image of a (111) nano-facet at (110) surface of ceria nanoparticle imaged in [110] projection at room temperature. Ce column is labeled as orange and oxygen column is labeled green. $b, c, d)$ Image sequence of the step edge surface with 130ms frames. The dynamic behavior of the Ce column has been highlighted by red arrow.

References

[1] Adler, S. B., Chemical Reviews, 104(2004), 4791-4844.

[2] Trovarelli, A., "Catalysis by ceria and related materials”(Imperial College Press, London), 25

[3] Mai, H., Journal of Physical Chemistry B, 109(2005), 24380-24385

[4] Lawrence, E., ACS Nano, 15(2021),2, 2624-2634

[5] Montini, T. Chemical Reviews, 116(2016), 5987-6041.

[6] The authors acknowledge funding from NSF DMR 1840841, NSF CBET 1604971 and the use of facilities of Eyring Materials Center at Arizona State University 